\title{
Pre-main sequence disks
}

\author{
Gaspard Duchêne $e^{1,2}$ \\ ${ }^{1}$ Astronomy Department, University of California Berkeley, Berkeley CA 94720-3411, USA \\ email: gduchene@berkeley.edu \\ ${ }^{2}$ Université Joseph Fourier - Grenoble 1/CNRS, Laboratoire d'Astrophysique de Grenoble \\ (LAOG) UMR 5571, BP 53, 38041 Grenoble Cedex 09, France
}

\begin{abstract}
In this contribution, I briefly review our empirical knowledge of disks around $\lesssim 2 M_{\odot}$ pre-main sequence (T Tauri) stars, focusing first on the dichotomic question of their frequency before moving on to some more detailed disk properties (overall orientation, total mass, outer radius). Finally, I conclude with a brief discussion of disks around embedded protostars, which will play in the next few years a major role in testing star formation theory and simulations.
\end{abstract}

Keywords. stars: formation, circumstellar matter, stars: pre-main sequence

\section{Introduction}

The study of disks around pre-main sequence stars is primarily motivated by our will to understand the initial conditions, processes and timescales associated with planet formation. However, it must be emphasized that the presence and detailed properties of these protoplanetary disks are determined during the star formation process itself, of which they are a natural by-product due to angular momentum conservation. The buildup of mass in a circumstellar disk is indeed set by the accretion history of the object, as well as by dynamical interactions with its immediate neighbours in the early phases of its evolution. Therefore, one can use circumstellar disks as a "final boundary condition" that must be met by star formation theories and simulations, akin to the roles of the IMF and stellar multiplicity (see contributions by J. Ascenso and H. Bouy, this volume). Below, I outline a few key findings that theory must account for.

\section{Frequency of disks around pre-main sequence stars}

For about three decades, the presence of circumstellar disks around T Tauri stars has been probed through their copious amounts of infrared excess, most recently through a slew of Spitzer surveys of nearby star-forming regions. Of particular interest for this discussion are studies of the youngest $(\lesssim 1 \mathrm{Myr})$ stellar populations, such as NGC 1333 and NGC 2024, where the proportion of members hosting a circumstellar disks is as high as $80 \%$. Even slightly older $(\sim 2 \mathrm{Myr})$ star-forming regions like Taurus-Auriga, the Trapezium Cluster and NGC 2068/71 boast disk frequency rates of $60 \%$ and higher (see Hernández et al. 2008 and references therein). Considering that some disks may be disrupted in early dynamical interactions between nearby objects, it is clear that the large majority ( $290 \%$ ) of stars form accompanied by a circumstellar disks.

Breaking down this global disk frequency, it is interesting to test whether the mass of the central star has any impact on the presence of a circumstellar disk, which could be expected via the depth of its potential well. Sufficiently large samples have been probed in several nearby star-forming regions (the Trapezium Cluster, Taurus-Auriga, IC 348, Chamaeleon I) so as to split them in a few stellar mass bins, with very similar results in 
each region (Hillenbrand et al. 1998; Luhman et al. 2010). In short, the disk frequency is typically only 20-30\% lower for very low-mass objects than for solar-type stars. Therefore, even among extremely low-mass objects, at least half are surrounded by a circumstellar disk at an age of $\sim 1$ Myr. We also note that there is no significant dependence of the disk frequency with stellar mass in the $0.25-2 M_{\odot}$. Unfortunately, there are too few Herbig AeBe stars (with masses in the $2-8 M_{\odot}$ range) known in star-forming regions to assess whether this high disk frequency extends to higher masses.

Another factor that is thought to play an important role in the detailed physics of star formation is the type of environment in which stars form. While young dense clusters are characterized by lower multiplicity rates thought to be caused by their violent early dynamical history, the four populations with disk frequencies higher than $80 \%$ are all young dense clusters (Hernández et al. 2008). It thus appears that disk formation and long-term survival are not nearly as strongly (if at all) affected in a densely clustered environment as multiplicity. The other way in which the environment can affect the formation and survival of circumstellar disks is through the intense UV radiation field imposed by high-mass O-type stars. However, in the Orion Trapezium Cluster, where a handful of high-mass stars are responsible for the well-known "proplyd" phenomenon, the proportion of young stars hosting a disk appears to be roughly constant, possibly even slightly decreasing, as a function of the distance to $\theta^{1} \mathrm{C}$ Ori on a scale of $1-3 \mathrm{pc}$ (Hillenbrand et al. 1998). This may be because the UV radiation field, while sufficient to photoevaporate the outer regions of disks, is nonetheless too weak to disrupt whole disks altogether. In any case, this seems to indicate that the ultraviolet field of high-mass stars does not preclude the formation of protoplanetary disks, except in the immediate vicinity of individual O stars (e.g., Guarcello et al. 2009, Mercer et al. 2009).

\section{Quantative properties of disks}

While assessing the presence of a disk around a pre-main sequence star is a relatively easy task, it is a more complex one to determine the disk bulk properties, such as total mass and global geometry. For one, near and mid-infrared observations only probe the presence of dust within a few AU of the central object. Generally speaking, going beyond this dichotomic assessment requires to make use of a combination of 1) simplistic assumptions, 2) complex radiative transfer modeling, and/or 3) spatially-resolved observations. As a result, using large populations of disks to test theoretical and numerical predictions is plagued by a complex set of biases and is generally a model-dependent endeavor.

Arguably the least ambiguous disk "property" to determine is the disk symmetry axis (i.e., the orientation of its angular momentum vector). Scattered light images and interferometric mapping at millimeter wavelengths have now successfully resolved tens of circumstellar disks in nearby star-forming regions $\dagger$, enabling global statistical studies. In particular, it is possible to compare the orientation of disks with respect to the local cloud magnetic field, as traced by linear polarization measurements of background stars. Contrary to earlier studies (that were plagued by small sample sizes and selection biases), it appears that the symmetry axis of young star+disk systems are randomly oriented with respect to the local magnetic field in the Taurus-Auriga cloud (Ménard \& Duchêne 2004). This suggests that the orientation of the initial magnetic field has little to no influence on that of the final angular momentum vector of the system, or, alternatively, that there is an almost complete "loss of memory" of any originally preferred orientation during the star formation process.

$\dagger$ See an up-to-date list at http://www.circumstellardisks.org 
The orientation of disks within multiple stellar systems is also an interesting tracer of the dynamical evolution inherent to the formation of the systems themselves. In binary systems, the circumstellar disks associated with each component appear to be preferentially aligned with each other, although there is a non-trivial number of exceptions (see Monin et al. 2007 for a review). Intriguingly, this is reminiscent of the relative orientation of orbital planes within triple systems (e.g., Tokovinin 1997). As for the relative orientation of disks and orbital planes, too few systems have been studied to date to reach definitive conclusions. It is worth noting, however, that there are examples of misalignment in the case of circumstellar disks (e.g., Stapelfeldt et al. 1998), i.e., disks around a given component, whereas circumbinary disks seem to be coplanar with their inner binary (e.g., Prato et al. 2002). Because the former type of systems is generally characterized by larger separations (a result of selection biases), this may be interpreted as evidence for a (partial) randomization of the individual angular momentum vectors during the fragmentation of a collapsing core.

Because disk studies have long focused on the question of planet formation, significant efforts have been placed on assessing their total mass and overall size, which are critical initial conditions. While there is a large object-to-object scatter and considerable uncertainty in the conversion of (sub)millimeter fluxes to total disk masses (due to unknown dust opacities and gas-to-dust ratios), the maximum disk masses over the $0.25-3 M_{\odot}$ stellar mass range is on the order of $10 \%$ of the stellar mass (see Natta et al. 2000 for a review). This conclusion seems to extend all the way into the brown dwarf regime (Bouy et al. 2008; Momose et al. 2010). This suggests that most, if not all, disks around pre-main sequence stars are intrinsically robust against gravitational instabilities. However, since disk instabilities occurs on much shorter timescales than the typical age of pre-main sequence stars, this should only be considered an inescapable conclusion. Disk fragmentation, if it occurs at all, must take place in a much earlier phase, with the presently observed disk a mere remnant of the initial disk or the result of subsequent continuous accretion from the collapsing core.

Finally, the outer radius of disks, which may be considered a fossil tracer of past dynamical evolution, remains difficult to assess with accuracy because of the limited spatial resolution and sensitivity to faint extended structures of current observations. Typical disk sizes are in the 50-500 AU range (Kitamura et al. 2002; Watson et al. 2007), with no significant correlation between disk size and stellar mass. Among disks resolved around single very low-mass stars, two are remarkably small (20-40 AU, Luhman et al. 2007; Momose et al. 2010) while one is extremely large (1100 AU, Glauser et al. 2008), suggesting that other factors are more important than the stellar mass in determining the disk size. Because we observe disks long after they form, the nature of these factors remains a speculative topic of discussion.

\section{The future: disks around embedded protostars}

Ultimately, the study of disk properties, even global ones like disk mass and size, in pre-main sequence stars is of little help to constrain the stellar formation process. While this is in part because of selection biases and instrumental limitations, there is a much more profound issue. By the pre-main sequence phase, a large fraction of the initial disk mass has been accreted or expelled from the system and viscous evolution has spread the disk's outer radius well beyond its original size. In order to use disk properties to test predictions of star formation theory, it is mandatory to move back in time and get as close as possible to star formation itself. Specifically, it is necessary to study disks around embedded protostars. 
Class I protostars represent the phase that precedes the T Tauri phase, and are a natural first step in that direction. Yet, only Class 0 sources, in which the central protostar still amounts for a minority of the total mass of the system, are readily comparable to the outcome of numerical simulations. Unfortunately, Class 0 sources are dominated by a massive envelope, and it is particularly challenging to discern their circumstellar disk. In dedicated studies, two remarkably large disks have been resolved and distinguished from the envelope in which they are embedded (150-300 AU, Jörgensen et al. 2005; Enoch et al. 2009). On the other hand, a first systematic survey of five several Class 0 has found no resolved disk on scales of 70-120 AU (Maury et al. 2010), suggesting either much smaller disk sizes or a delayed formation of disks. The picture is still murky at this point, but further studies of disks among Class 0 sources in the upcoming $A L M A$ era will yield a clearer picture and, consequently, a more stringent test of star formation theories and simulations.

\section{References}

Bouy, H., Huélamo, N., Pinte, C., Olofsson, J., Barrado y Navascués, D., Martín, E., Pantin, E., Monin, J. L., Basri, G., Augereau, J. C., Ménard, F., Duvert, G., Duchêne, G., Marchis, F., Bayo, A, Bottinelli, S., lefort, B., \& Guieu, S. 2008, A\& A, 486, 877

Enoch, M., Corder, S., Dunham, M., \& Duchêne, G. 2009, ApJ, 707, 103

Glauser, A., Ménard, F., Pinte, C., Duchêne, G., Güdel, M., Monin, J. L., \& Padgett, D. 2008, $A \mathscr{E} A, 485,531$

Guarcello, M., Micela, G., Damiani, F., Peres, G., Prisinzano, L., \& Sciortino, S. 2009, A\&̇A , 496,453

Hernández, J., Hartmann, L., Calvet, N., Jeffries, R., Gutermuth, R., Muzerolle, J., \& Stauffer, J. 2008, ApJ, 686, 1195

Hillenbrand, L., Strom, S., Calvet, N., Merril, M., Gatley, I., Makidon, R., Meyer, M., \& Skutskie, M. $1998, A J, 116,1816$

Jörgensen, J., Bourke, T., Myers, P., Schöier, F., van Dishoeck, E., \& Wilner, D. 2005, ApJ, 632,973

Kitamura, Y., Momose, M., Yokogawa, S., Kawabe, R., Tamura, M., \& Ida, S. 2002, ApJ, 581, 357

Luhman, K., Allen, P., Espaillat, C., Hartmann, L., \& Calvet, N. 2010, ApJS, 186, 111

Luhman, K., Adame, M., D'Alessio, P., Calvet, N., McLeod, K., Bohac, C., Forrest, W., Hartmann, L., Sargent, B., \& Watson, D. 2007, ApJ, 666, 1219

Maury, A., André, P., Hennebelle, P., Motte, F., Stamatellos, D., Bate, M., Belloche, A., Duchêne, G., \& Whitworth, A. 2010, $A \& A$ A, 512, 40

Ménard, F. \& Duchêne, G. 2004, A\& A, 425, 973

Mercer, E., Miller, J., Calvet, N., Hartmann, L., Hernández, J., Sicilia-Aguilar, A., \& Gutermuth, R. 2009, ApJ, 138, 7

Momose, M., Ohashi, N., Kudo, T., Tamura, M., \& Kitamura, Y. 2010, ApJ, 712, 397

Monin, J. L., Clarke, C., Prato, L., \& McCabe, C. 2007, in: B. Reipurth, D. Jewitt, \& K. Keil (eds.), Protostars 8 Planets $V$, p. 395

Natta, A., Grinin, V., \& Mannings, V. 2000, in: V. Mannings, A. Boss, \& S. Russell (eds.), Protostars 85 Planets $I V$, p. 559

Prato, L., Simon, M., Mazeh, T., Zucker, S., \& McLean, I. 2002, ApJ (Letters), 579, L99

Stapelfeldt, K., Krist, J., Ménard, F., Bouvier, J., Padgett, D., \& Burrows, C. 1998, ApJ (Letters), 502, L65

Tokovinin, A. 1997, A\&AS, 124, 75

Watson, A., Stapelfeldt, K., Wood, K., \& Ménard, F. 2007, in: B. Reipurth, D. Jewitt, \& K. Keil (eds.), Protostars 8 Planets V, p. 523 